\title{
Sciendo
}

ACTA UNIVERSITATIS CIBINIENSIS - TECHNICAL SERIES

DOI: $10.2478 /$ aucts-2019-0010

Vol. 712019

\section{CUSTOMIZABLE PATIENT-SPECIFIC CUTTING GUIDE FOR HIGH TIBIAL OSTEOTOMY}

\author{
PETRUSE Radu Emanuil \\ Faculty of Engineering, Department of Industrial Engineering and Management, "Lucian Blaga" \\ University,Sibiu, Romania, radu.petruse@ulbsibiu.ro \\ COFARU Nicolae Florin \\ Faculty of Engineering, Department of Industrial Engineering and Management, "Lucian Blaga" \\ University, Sibiu, Romania, nicolae.cofaru@ulbsibiu.ro \\ COFARU Ileana Ioana \\ Faculty of Engineering, Department of Computer Science and Electrical Engineering, "Lucian Blaga" \\ University, Sibiu, Romania, ioana.cofaru@ulbsibiu.ro
}

\begin{abstract}
The paper is an interdisciplinary medical and engineering approach that aims to optimize the accuracy of the High Tibial Osteotomy surgical intervention. The axial deviations of the human inferior limb are presented in the first part of the paper. Without altering the shape of the bones, these deviations cause the wear of the knee's articular cartilage by gonarthrosis. One of the most common and effective treatment modalities for eliminating axial deviations is High Tibial Osteotomy. Because the surgical procedure involves $3 D$ spatial bone cutting, the accuracy of the procedure and thus the success of the operation and the recovery of the patient is dependent on the execution of these cuts. Within this paper, a specialized device is proposed that makes it possible to guide the surgical saw and to accurately perform the correction angles. In the last part of the paper, customization possibilities and fabrication of the main elements of the cutting device using Additive Manufacturing processes are presented.
\end{abstract}

Key words: High Tibial Osteotomy, Customized cutting guide

\section{Introduction}

Nowadays, a considerable part of the population is affected by a quite common disorder, osteoarthritis, which at the knee level is also called gonarthrosis. This disease is due to the wearing and the decrease of the resistance of the intra-articular cartilage at the level of the knee which automatically leads to a defective mechanical loading in the joint. This causes an axial deviation to the level of the lower limb, implying that the mechanical axis of the leg joining the centre of the femoral head and the middle of the ankle (purple line from figure 1) will no longer pass through the centre of the knee but medially or laterally resulting in the conditions known as varum or valgus genu. (Figure 1) 

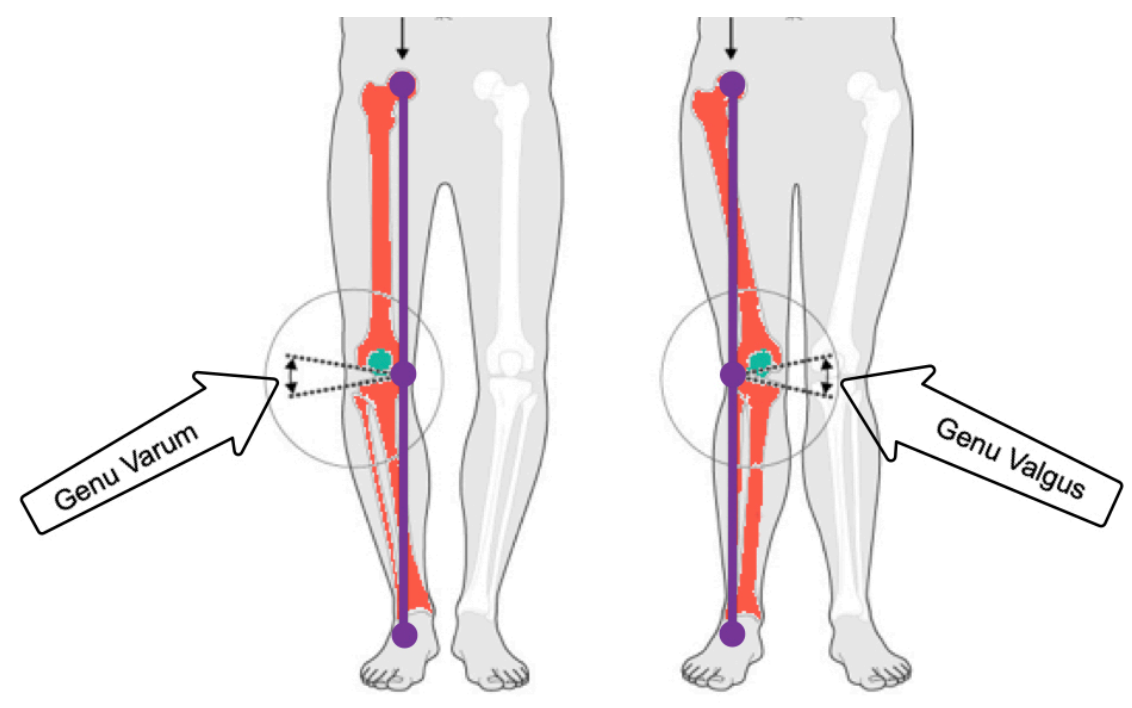

Figure 1 Axial deviations as seen from the frontal plane [1]

Gonarthrosis causes axial deflections which result in higher loads and with greater intensities on the knee, due to the incorrect knee loads. By amplifying the gonarthrosis appears a vicious circle in which the two effects are mutually potentiated.

This vicious circle has to be broken and one of the most effective, applicable and recommended variants is the High Tibial Osteotomy (HTO) (Figure 2).

This procedure implies the creation of a wedge in the bone at the level of proximal human tibia in order to eliminate the axial deviations and to realign the mechanical axis of the human lower member with the possibilities presented in figure 2.

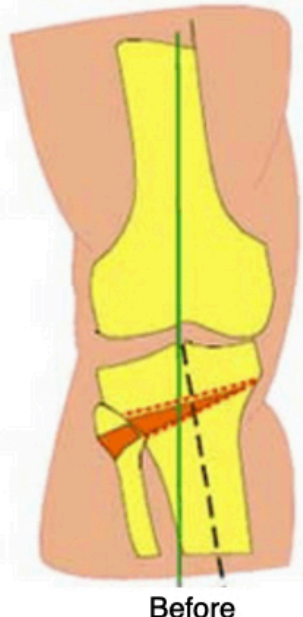

Before

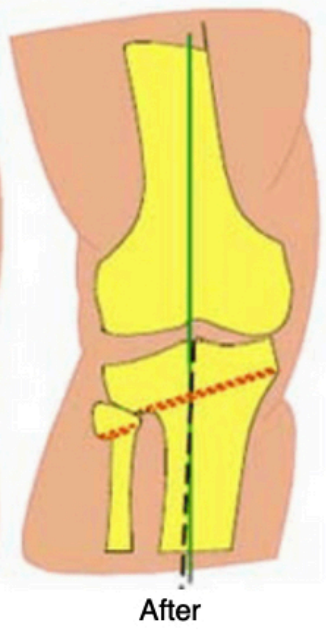

Closing-wedge high tibial osteotomy (OW-HTO)

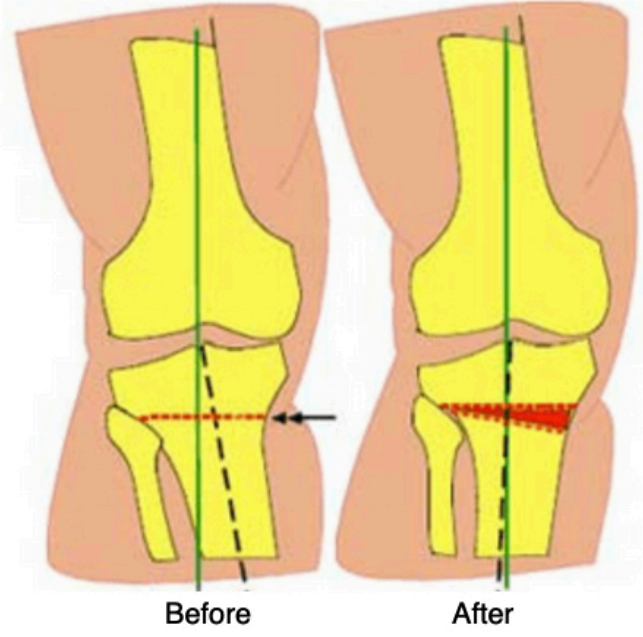

Opening-wedge high tibial osteotomy (OW-HTO)

Figure 2 High tibial osteotomy type [2]

For a successful surgical intervention is very important to establish as accurate as possible the hinge point in frontal plane. This point is named CORA (Centre of Rotation of Angulation). Also, to perform a hole form stress relief in this point and obviously to execute a precise angular cut. These things make the difference in a successful HTO and for this the dexterity of the surgeon is not enough, a specialized device is required [3].

\section{Customizable patient-specific cutting guide for HTO}

A precise angular cut makes the difference in a successful HTO. In order to achieve this, an angular correction must be done in three spatial planes. Patient-specific cutting guides (PSCG) is one of the instruments developed to aid the surgeons in obtaining a reliable and accurate correction [4]. Within this 
research we propose creating 3D printed PSCG for opening-wedge high tibial osteotomy (OW-HTO) and for closing-wedge high tibial osteotomy (CW-HTO).

\subsection{Designing the PTSG}

The first phase in developing a 3D printed PSCG is to obtain a CAD model of the patient's tibia or, if not possible the dimensions from an $2 \mathrm{D} x$-ray. The CAD model is created from a preoperatory computed tomography (CT) scan. DICOM files obtained from CT are imported into an open source software "Slicer" where the required texture can be isolated and transformed into 3D volumes. In our case we have isolated the bone tissue of the tibia, refined it and converted it into a 3D model which can be used in a CAD software. On this CAD model the PSCG is virtually positioned and customized accordingly to the surgeon's specifications. Where a CT scan is not available, a simple 2D x-ray of the patient's tibia can be used to determine the main dimensions required to personalize a parameterized 3D CAD model of a generic tibia. For this research purposes we used the Slicer software to convert the DICOM files to 3D CAD models. Afterwards, the resulted 3D model of the tibia is imported in Catia, a CAD software, where a virtual surgery planification is performed.

The first step is to position the general elements of the cutting guide in the right position. This procedure is calculated based on the CORA point and the correction angles, as it can be seen in figure 3 . First the cutting guide fixture (yellow part) is placed into the right position with a device that uses the CORA point as reference. After this precise positioning the cutting guide fixture is fixed into position with two screws.
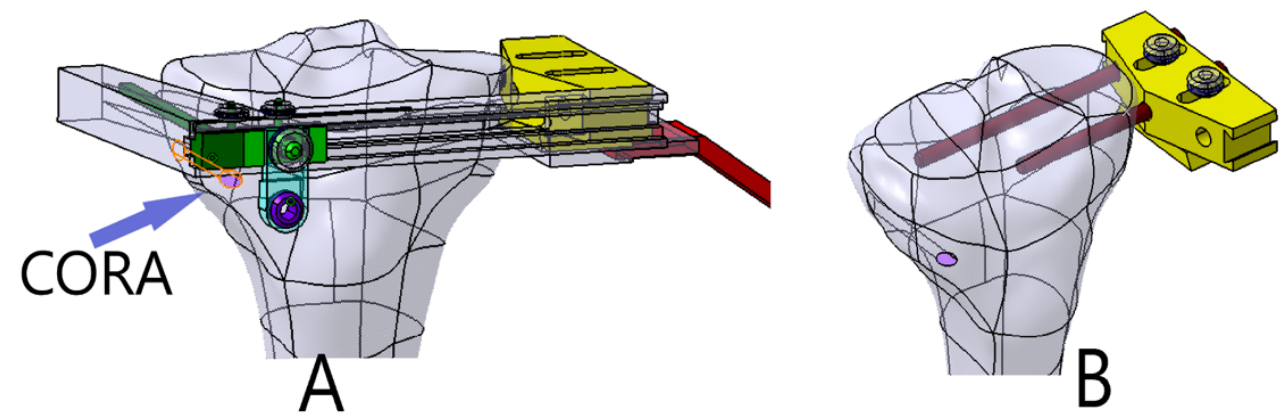

Figure 3 Positioning the cutting guide fixture

The PSCG can now be attached to the cutting guide fixture and it can be customized according to the osteotomy correction required. The cutting guide is designed to aid multiple types of HTOs (OW-HTO, $\mathrm{CW}-\mathrm{HTO}$ and biplanar HTO) as depicted in figure 4. The only modification required to the other components of the device is to shorten the cutting guide fixture when it is used for a biplanar HTO. In this case an extra part is required to be able to use the same positioning device presented in figure 5 .

In figures 5 and 6 the assembled device can be observed, customised to aid OW-HTO, CW-HTO and biplanar HTO.

The most important part of the PTSG, the cutting guide, presented in figure 4 offers a variable correction angle. In order to increase even more the HTO precision we propose to create a patient-specific cutting guide onto which the correction angle is specified exactly to the patient's needs. In order to be able to fabricate this component in a timely manner, we propose using Additive Manufacturing processes. 


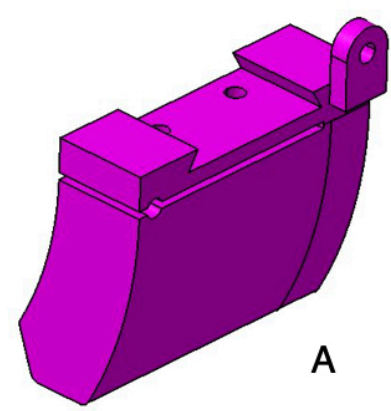

OW-HTO

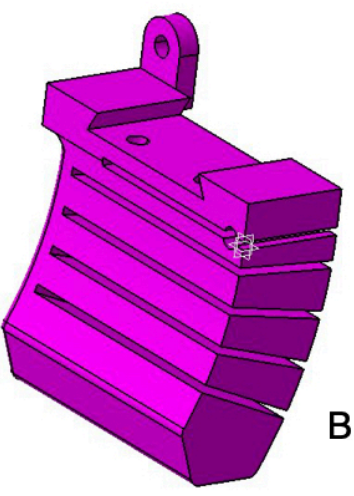

CW-HTO

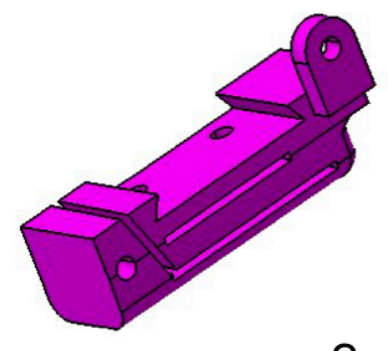

C

Biplanar-HTO

Figure 4 Customized cutting guides for different types of the osteotomies
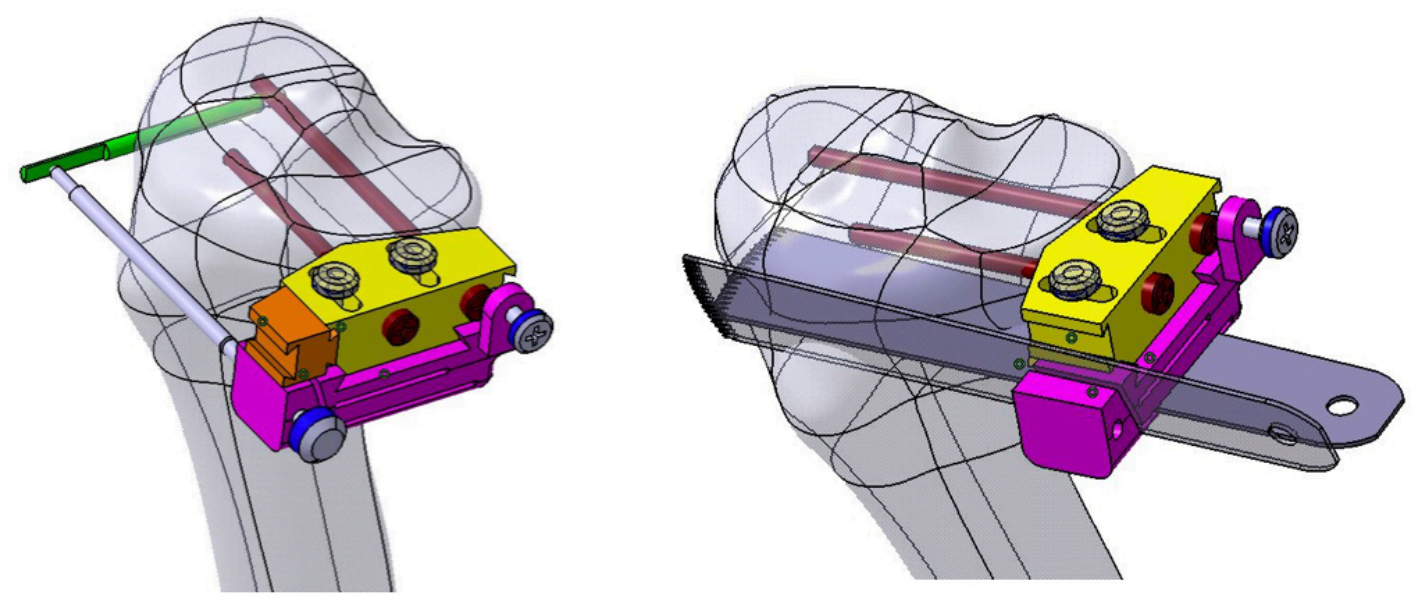

Figure 5 Variable cutting guide fixture for biplanar HTO
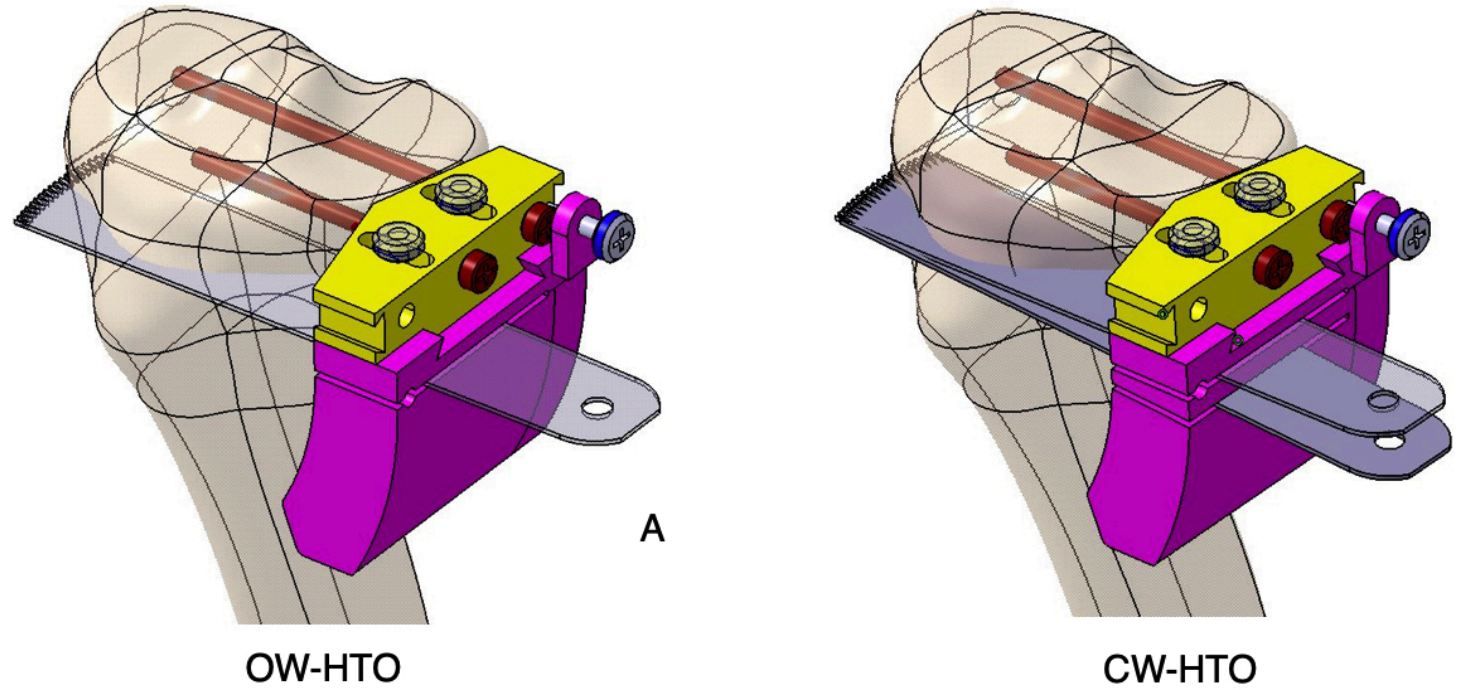

B

Figure 6 HTO possibilities with the customized PSCG

Beside the geometrical customizations of the PSCG we also included some parameterized dimensions which will aid the personalization process. With these parameters we can easily modify the angles for the cutting guidance slots based on the patient's requirements and we are able to vary the geometry for biplanar HTO (figure 7). 

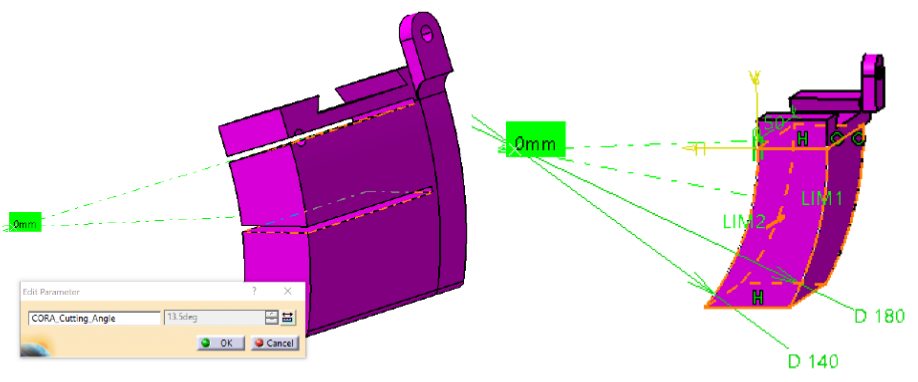

A-CORA cutting angle

B-CORA based diameter

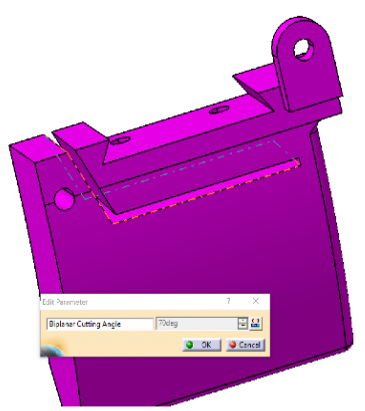

C-Biplanar angle

Figure 7 Customization parameters

\subsection{Fabrication of the PTSG}

The most suitable fabrication method for our PTSG is additive manufacturing. We chose this process due to the advantages it presents in terms of production time of highly personalized components. As an additive manufacturing process, we chose fused deposition modelling (FDM) since we have previous experience with this process and how to improve the physical properties of the resulted parts [5].

The parts were fabricated on a Ultimaker3 3D printer which uses Cura version 3.3.1 printing software to manage the printing process. The printing parameters that mostly influence the physical properties of the printed parts were chosen as follows: Build orientation (Bo) vertical; Infill density (Id) 70\%; Infill pattern X; Raster angle (Ra) 45 ; Side wall thickness (Vt) 1,5mm; Layer thickness (Lt) 0,2mm; Printing speed (Ps) $60 \mathrm{~mm} / \mathrm{s}$; Printing temperature (T) $240^{\circ} \mathrm{C}$; Nozzle diameter (NØ) $0,4 \mathrm{~mm}$. The material used for this experiment is acrylonitrile-butadiene-styrene (ABS) that comes as $1.75 \mathrm{~mm}$ filament.

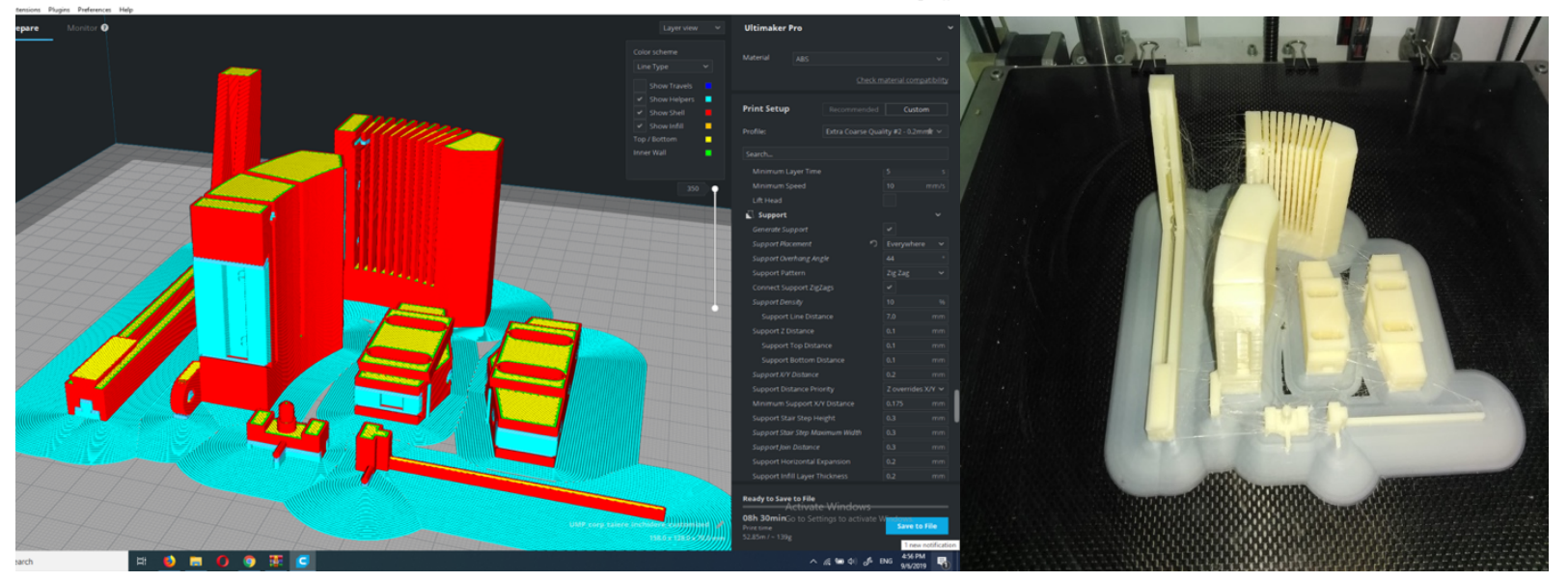

Figure 8 3D printing preparation and results

These 3D printed PSCG present several advantages over conventional modular PSCG. The ones worth mentioning are:

- $\quad$ geometry and shape are easily adaptable for patient-specific requirements;

- components design to fabrication time is diminished due to CAD-3D printer file compatibilities, thus avoiding the use of additional processes (e.g. NC code generation for milling machines);

- $\quad$ components can be fabricated in a sterile environment;

- $\quad$ sterilization is possible using autoclaves at around $120^{\circ} \mathrm{C}$

- $\quad$ after use the components can be easily disposed and recycled.

\section{Conclusions}

Nowadays due to a general lack in sports activities and with an often-unhealthy diet, many people are prone to obesity of different degrees that has direct consequences on the loads in the articular zones, especially in the case of the knees. This is why gonarthrosis with axial deviations of the lower limbs has become quite common in younger subjects as well. A surgical procedure especially applicable to younger patients is HTO. The precision of this operation is very important in the success of the surgery and the recovery of the patient. Therefore, the dexterity of the surgeon should not be the main variable anymore. In 
this regard, we propose a PSCG that pinpoints the exact location of the CORA hinge, aids the creation of a hole to relief the strain and guides the surgeon precise in performing the necessary cuts. The proposed device has a modular design which is also parameterized so that it can be adaptable to any type of HTO. More cutting guides are designed in such a way as to allow the adjustment of the angles of cuts continuously or in steps and even with the possibility of concrete particularization for a given patient. The fabrication of the main component of the device, the cutting guide, which is patient-specific can be obtained by Additive Manufacturing processes. Moreover, the personalized cutting guide can be sterilized in autoclaves and it can be easily recycled after the surgery.

\section{Acknowledgment}

This work is supported through the DiFiCIL project (contract no. 69/08.09.2016, ID P_37_771) cofounded by ERDF through the Competitiveness Operational Programme 2014-2020.

\section{References}

1. J. E. Carreiro, "Lower leg," in Pediatric Manual Medicine, Churchill Livingstone , 2009, p. 273-327.

2. Medifee," [Online]. Available: https://www.medifee.com/surgery/knee-osteotomy-in-india. [Accessed 09 2019].

3. Ileana Ioana COFARU - Experimental modular stand used for studies of the High Tibial Osteotomy, MATEC Web Conf, Volume 121, 2017

4. Chaouche, S., Jacquet, C., Fabre-Aubrespy, M. et al. International Orthopaedics (SICOT) (2019).

5. R. E. Petruse, S. Pușcașu, A. Pascu, I. Bondrea. Materials Today: Proceedings Volume 12, Part 2, 2019, pp. 358-366 